\title{
Aggregate versus Excimer Emissions from Poly(2,5-di-n-octyloxy-1,4-phenylenevinylene)
}

\author{
Y. F. Huang, ${ }^{\dagger}$ Y. J. Shiu, ${ }^{\dagger}$ J. H. Hsu, ${ }^{\ddagger}$ S. H. Lin,${ }^{\dagger}$ A. C. Su, ${ }^{\ddagger}, \mathbb{I}$ K. Y. Peng,, \\ S. A. Chen," and W. S. Fann $*,+, s$ \\ Institute of Atomic and Molecular Sciences, Academia Sinica, P.O. Box 23-166, Taipei 106, Taiwan, \\ Department of Materials Science and Optoelectronic Engineering, National Sun Yat-sen University, Kaohsiung \\ 804, Taiwan, Department of Physics and Institute of Polymer Science and Engineering, National Taiwan \\ University, Taipei 106, Taiwan, and Department of Chemical Engineering, National Tsing-Hua University, \\ Hsinchu 300, Taiwan
}

Received: November 22, 2006; In Final Form: February 7, 2007

\begin{abstract}
Using fractions of poly(2,5-di-n-octyloxy-1,4-phenylenevinylene) (DO-PPV) of different chain lengths, the interchain interactions in both the solution and the bulk states from ambient to cryogenic temperatures were examined via a combination of steady-state and time-resolved photoluminescence spectroscopic techniques. Results indicated that the emission from these aggregates bears characteristics of quantum mechanical dimerization, not that of the extended conjugation of single chains. The emission spectrum from films is generally consistent with the "aggregate emission" spectrum extracted from aggregated solutions; nevertheless, there indeed exists minor emission at the red end of the spectrum, representing contribution from excimers.
\end{abstract}

\section{Introduction}

Since the discovery of the light-emitting polymer, considerable efforts have been made toward understanding its electronic properties. $^{1-22}$ One of the issues related for these materials in the solid state is the role of interchain interactions..$^{23-26}$ In general, there are two categories of the interchain effects upon ordered packing of conjugated chains, namely, the morphological change and the formation of the interchain electronic states. The former leads to an increase of conjugation length via improved backbone coplanarity, ${ }^{27,28}$ whereas the latter causes the delocalization of excitations over different chains; $;^{29-38}$ the latter includes both aggregate and excimer emissions. Through their weak interaction, two neighboring chains may form a bound system as a dimer, the simplest form of aggregates. This aggregate state is usually characterized by changes in absorbance and fluorescence spectra. An alternative interchain state, that is, an excimer, is formed by the interaction between an excited chain with a ground-state neighbor. The primary spectral feature of such a state is that the absorbance spectrum does not change significantly (as compared with the intrachain state), but the corresponding emission spectrum is significantly red-shifted, becoming structureless.

Bässler et al. ${ }^{31}$ investigated ladder-type poly( $p$-phenylene) (LPPP) and showed that both absorbance and photoluminescence (PL) spectra of LPPP thin films acquire an additional low-energy band as compared to that of the solution-state counterparts. They ascribed this to the existence of aggregates, instead of excimers, in the LPPP film. However, Samuel et al. ${ }^{32-34}$ found that PL spectra of cyano-grafted poly(phenylenevinylene) (CN-PPV) thin films are red-shifted, broad, and structureless as compared to those from the solution phase; the absorbance spectra of both phases are similar. Model calculations by Conwell et al. ${ }^{9}$ lent further support to the existence of excimer states in $\mathrm{CN}-\mathrm{PPV}$

\footnotetext{
Academia Sinica.

$\doteqdot$ National Sun Yat-sen University.

$\S$ National Taiwan University.

II National Tsing-Hua University.
}

films, which is consistent with the observation of a relatively long lifetime, approximately $5 \mathrm{~ns}$. Similar spectral characteristics were identified ${ }^{39}$ for the excimer state of a related polymer, poly(2,5-di(2'-methylhexyloxy)-1,4-phenylenevinylene) (MEH-PPV), with an extended lifetime of $450 \mathrm{~ns}$ at the cryogenic temperature of study.

The interchain interactions can be sensitive to slight changes of the side-chain structure. It was reported ${ }^{40}$ that formation of aggregates can be readily observed in all-trans-2,5-diheptyloxy$p$-divinylbenzene. In the case of 2,5-diheptyl-p-divinylbenzene (i.e., in the absence of the ether linkage), aggregate formation is suppressed. ${ }^{41}$ Another example can be drawn from $\mathrm{MEH}-$ PPV and its close homologue poly(2,5-di- $n$-octyl-1,4-phenylenevinylene) (DO-PPV); with the slight change from branched to linear side-chains (with carbon number fixed), DO-PPV shows much stronger tendency toward aggregation.

Here, we report a spectroscopic study of two DO-PPV fractions of contour lengths of $\sim 6$ and $\sim 11 \mathrm{~nm}$, which are shorter than and comparable to the persistence length of DOPPV, respectively. For a close homologue of DO-PPV, poly[2-butoxy-5-(2'-ethylhexyloxy-1,4-phenylenevinylene)] (BEHPPV), the persistence length has been determined via light scattering to be ca. $11 \mathrm{~nm} .{ }^{27}$ We find that the interchain interaction is very sensitive to this change in chain length, which allows for resolution and identification of different excitonic species. Our results are generally in favor of emission from delocalized interchain species (as compared to single-chromophore emission with extended conjugation due to improved backbone coplanarity), especially the aggregate mechanism. Contributions from excimers at the red end of the spectrum can be identified in the film state, but these are comparatively minor.

\section{Experimental Methods}

Materials. DO-PPV is prepared following a procedure similar to that adopted by Sarnecki et al. ${ }^{42}$ and Askari et al. ${ }^{43}$ The weight-average molecular mass of the nascent sample was $2 \mathrm{MDa}$ with polydispersity greater than 5 , as determined by 
gel permeation chromatography (GPC) using polystyrene standards. This sample was fractionated using the procedure described previously. ${ }^{44}$ Two fractions of DO-PPV, with $\mathrm{M}_{\mathrm{w}}$ $=8.8$ and $16 \mathrm{kDa}$, were used for the present study; the corresponding polydispersity values were 1.53 and 2.10 , respectively. Note that these molecular mass values are polystyreneequivalent in terms of GPC elution time and, hence, are used here as nominal values; the true values are likely lower by a factor of ca. $3 .{ }^{45}$ A good solvent, chloroform, and a relatively poor solvent, 2-methyltetrahydrofuran (m-THF), were chosen for this work. Solutions were prepared by first dissolving the samples in chloroform, followed by dilution in $\mathrm{m}$-THF. The $\mathrm{m}$-THF solutions were routinely warmed to $60{ }^{\circ} \mathrm{C}$ (to ensure dissolution) and then allowed to cool to ambient temperature before any measurements. For low-temperature ( $77 \mathrm{~K})$ measurements, further cooling of the m-THF solution was done through a flash-freeze procedure. The concentrations of both the lowand high-molecular weight solutions are $\mathrm{C} 0=10^{-6}, \mathrm{C} 1=10^{-5}$, and $\mathrm{C} 2=10^{-4} \mathrm{M}$. The thin films were spin cast from the solution with a concentration of $5 \mathrm{mg} / \mathrm{mL}$.

Instrumentation. Optical absorbance spectra of the sample solutions were obtained using a GBC Cintra-20 instrument, in which the uncertainty of the spectrometer is OD $\sim 0.01$. The fluorescent (PL) and excitation (PLE) spectra were recorded either by a liquid nitrogen-cooled, CCD-based system or a Jobin Yvon FL3-21 spectrofluorometer. The bandwidths are $1 \mathrm{~nm}$ for both excitation source and emission measurements, and the instrumental response has been corrected. All room experiments were performed in a $1 \mathrm{~cm}$ cuvette. Fluorescence spectra were taken in front-face geometry to minimize self-absorption at high concentrations. We performed high-concentration experiment in good solvent to ensure negligible self-absorption and inner filter-induced spectral distortion. Time-resolved fluorescence spectra were obtained by a time-correlated single-photon counting system (TCSPC, Fluo Time 200, PicoQuant Inc.). The excitation source was a 100 fs tunable optical parametric amplifier (OPA 9400, Coherent, Inc.) with a repetition rate of $20 \mathrm{kHz}$. Here, the OPA laser was generated by an amplifier of a Ti-sapphire laser system (RegA 9000, Coherent, Inc.), which was pumped and seeded by an argon ion laser (Innova 400, Coherent, Inc.) and an oscillator of the $\mathrm{Ti}$-sapphire laser system (Mira 900, Coherent, Inc.). With a pump power density of $10^{3} \mathrm{~mW} \mathrm{~cm}^{-2}$, the sample solution was excited by the OPA laser at 500 or $560 \mathrm{~nm}$. Fluorescence signals probed at the range of 575-625 nm were collected at a right angle to the sample cuvette and collimated with a pair of focus lenses. With a GlanThomson polarizer and a double monochromator with dispersion subtraction, the fluorescence signals were recorded by an MCPPMT (R3809U-51, Hamamatsu) and collected by use of a data acquisition board (SPC630, Becker \& Hickle). The fwhm of the instrument response function (IRF) of the TCSPC system is about 40 ps. For thin films, low-temperature measurements were performed in a closed cycle refrigerator using a frequencydouble mode-locked $\mathrm{Ti}$-sapphire operated at $450 \mathrm{~nm}$, from which spectral dynamics in the nanosecond scale can be resolved. The size of aggregates in solutions was determined by means of dynamic light scattering using a Malvern PCS100 instrument $(\mathrm{He}-\mathrm{Ne}$ laser incidence beam; detector positioned at an angle of $90^{\circ}$ to incidence). For each sample, 10 measurements were made, and the average value of the $\mathrm{z}$-average hydrodynamic radius is reported here; the attached error bounds correspond to the standard deviations.

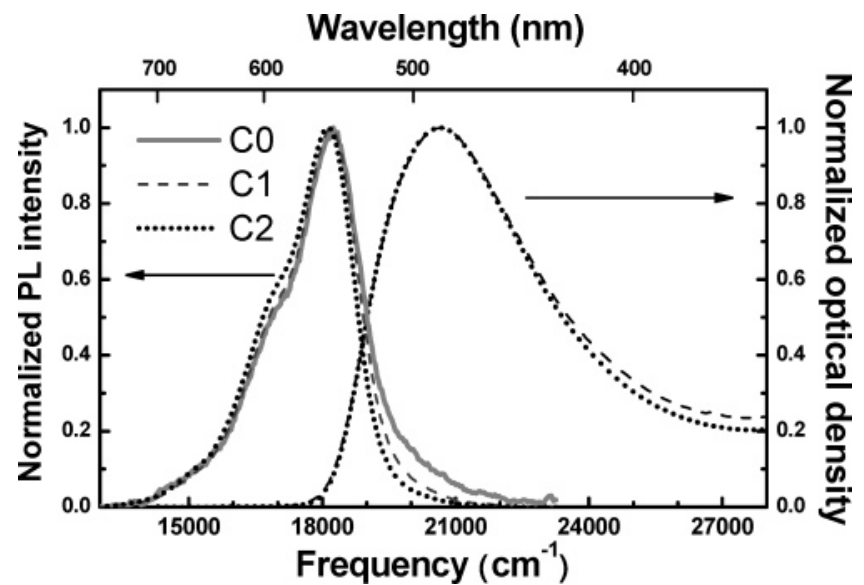

Figure 1. Normalized absorbance and PL $\left(\lambda_{\mathrm{ex}}=420 \mathrm{~nm}\right)$ spectra of the high molecular mass $\left(M_{\mathrm{w}} \sim 16 \mathrm{kDa}\right) \mathrm{DO}-\mathrm{PPV}$ in chloroform with concentrations (in monomer molarity) of $\mathrm{C} 0=10^{-6}, \mathrm{C} 1=10^{-5}$, and $\mathrm{C} 2=10^{-4} \mathrm{M}$.

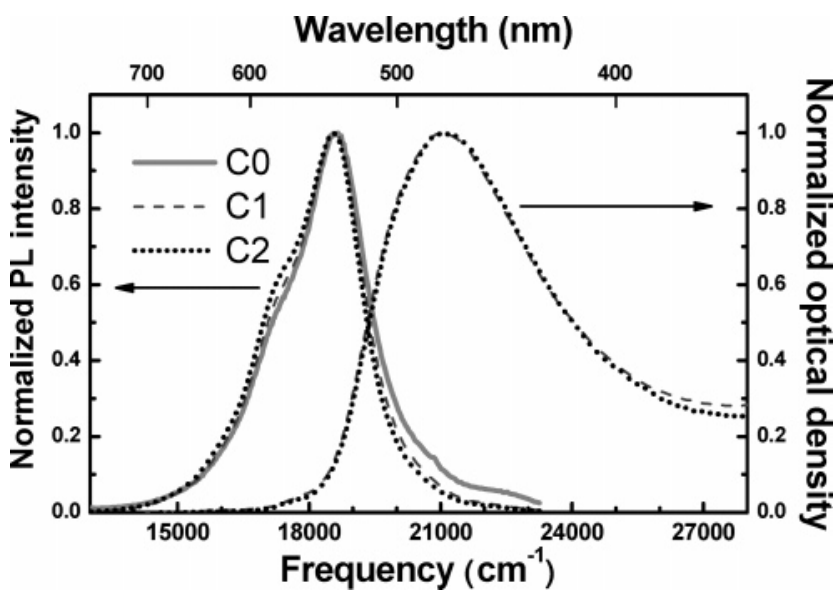

Figure 2. Normalized absorbance and PL $\left(\lambda_{\mathrm{ex}}=420 \mathrm{~nm}\right)$ spectra of the low molecular mass $\left(M_{\mathrm{w}} \sim 9 \mathrm{kDa}\right) \mathrm{DO}-\mathrm{PPV}$ in m-THF with concentrations of $\mathrm{C} 0=10^{-6}, \mathrm{C} 1=10^{-5}$, and $\mathrm{C} 2=10^{-4} \mathrm{M}$.

\section{Results and Discussion}

Single-Chromophore Emission. Figure 1 shows the absorbance and PL spectra (excited at $420 \mathrm{~nm}$ ) of the high molecular mass, $16 \mathrm{kDa}$ DO-PPV in chloroform with concentrations of $\mathrm{C} 0=10^{-6}, \mathrm{C} 1=10^{-5}$, and $\mathrm{C} 2=10^{-4} \mathrm{M}$. Note that the maximum absorbance was $\sim 0.2$ for $\mathrm{C} 1$ and 1.5 for $\mathrm{C} 2$; the absorbance spectrum for $\mathrm{C} 0$ is not shown as the noise level was comparable to spectroscopic readings in this case of extreme dilution. These spectra are clearly independent of solution concentration, with the emission maximum located at 18200 $\mathrm{cm}^{-1}$ and the maximum absorbance located at $21000 \mathrm{~cm}^{-1}$. As chloroform is a good solvent for DO-PPV, we conclude that the emission spectrum here corresponds to single-chain emission even at the highest concentration of $10^{-4} \mathrm{M}$ (which translates to ca. $0.36 \mathrm{w} / \mathrm{v} \%)$. Given in Figure 2 are absorbance and PL spectra (excited at $420 \mathrm{~nm}$ ) for the $9 \mathrm{kDa} \mathrm{DO}-\mathrm{PPV}$ in poor solvent $\mathrm{m}-\mathrm{THF}$. We note that the PL spectra here are very similar to those in Figure 1 except for the red shift by ca. 500 $\mathrm{cm}^{-1}$ (the peak position of the m-THF solution is $18700 \mathrm{~cm}^{-1}$ ) due to the difference between the dielectric constants of chloroform and m-THF. The PL spectra of $9 \mathrm{kDa}$ DO-PPV in both $\mathrm{m}$-THF and chloroform are almost identical. There exists a slight spectrum shift due to the dielectric constant difference. In other words, single-chain emission is the dominant mode for both $9 \mathrm{kDa}$ DO-PPV in $\mathrm{m}-\mathrm{THF}$ and $16 \mathrm{kDa}$ DO-PPV in chloroform. 

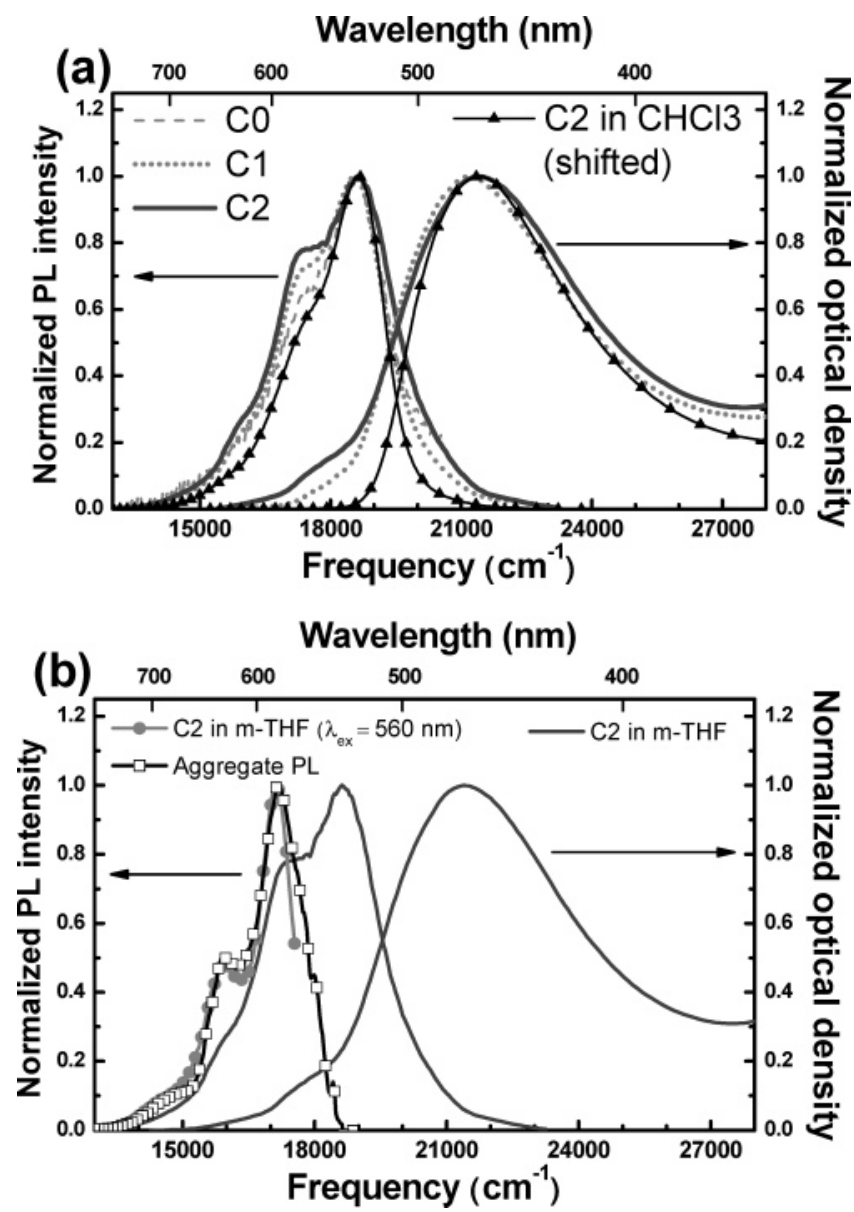

Figure 3. (a) Absorbance and PL $\left(\lambda_{\mathrm{ex}}=420 \mathrm{~nm}\right.$ unless specified otherwise) spectra of $16 \mathrm{kDa}$ DO-PPV in chloroform (solid line with filled triangles, horizontally shifted to match the intensity maxima of the m-THF solutions) and m-THF at concentrations of $\mathrm{C} 0=10^{-6}$ (dashed line), $\mathrm{C} 1=10^{-5}$ (dotted line), and $\mathrm{C} 2=10^{-4} \mathrm{M}$ (solid line). (b) The solid line with filled circles is the PL spectrum of m-THF solution with $\lambda_{\mathrm{ex}}=560 \mathrm{~nm}$. The solid line with open squares is the difference spectrum of the corresponding PL spectra from m-THF and chloroform solutions (with the latter horizontally shifted to match the intensity maxima).

Aggregation in m-THF. Figure 3 a shows the absorbance as well as the PL spectra (excited at $420 \mathrm{~nm}$ ) of the high molecular mass $16 \mathrm{kDa} \mathrm{DO}-\mathrm{PPV}$ in $\mathrm{m}$-THF with the same concentration range as that in Figure 1. Results of the high molecular mass DO-PPV in poor solvent were totally different from those of the low molecular mass case. In contrast to Figure 2, both the absorbance and PL spectra change shapes as the concentrations vary in high molecular weight samples. This indicates that there are additional species participating in the absorbance and emission other than the single-chain excitons. The solid lines shown in Figure $3 b$ are the absorbance and PL spectra (excited at $420 \mathrm{~nm}$ ) of $16 \mathrm{kDa}$ DO-PPV in m-THF at a higher concentration, $\mathrm{C} 2$. In addition to the broad absorbance maximum at $21500 \mathrm{~cm}^{-1}$, there emerges a shoulder at $18000 \mathrm{~cm}^{-1}(\sim 560$ $\mathrm{nm})$. The PL spectrum changes dramatically upon an increase in concentration to $\mathrm{C} 2$ for $16 \mathrm{kDa} \mathrm{DO}-\mathrm{PPV}$ in $\mathrm{m}-\mathrm{THF}$. As the concentration increases, the relative intensity of the highfrequency emission decreases, and a newly formed lowfrequency emission band (with the peak position lower by 1300 $\mathrm{cm}^{-1}$ ) develops. Note that the solid line with the open squares is the difference spectrum for the m-THF and the chloroform (as a single-chromophore reference) solutions obtained by horizontally shifting the spectrum of the chloroform solution to have its first peak overlapped with that of the m-THF solution.
This is taken to represent the interchain emission spectrum, as fluorescence spectra at all three concentrations can be modeled by assuming coexistence of intra- and interchain excitons. ${ }^{46}$ One may further observe from Figure $3 b$ that this difference spectrum coincides almost perfectly with the PL spectrum of the C2 solution in m-THF excited at $560 \mathrm{~nm}$ (the position of the additional absorbance shoulder), indicating they are of the same origin, interchain species. Because the species exist both in absorbance (ground state) and PL spectra (excited state), the spectrum is attributed to aggregates. Accordingly, the PL spectrum of $16 \mathrm{kDa}$ DO-PPV in m-THF (solid line in Figure 3 b, excited at $420 \mathrm{~nm}$ ) comprises both single-chromophore and aggregate emissions. The additional absorbance shoulder at 560 $\mathrm{nm}$ of the m-THF solution (as compared to that of the chloroform solution) is then assigned to aggregates absorbance.

Note that we cannot obtain the aggregated emission spectrum shown in Figure $3 \mathrm{~b}$ by subtracting high concentration data by the low concentration data shown in Figure 3a. Comparing the lowest concentration $\mathrm{C} 0$ of the m-THF solution with the $\mathrm{C} 2$ concentration in chloroform (the spectra are horizontally shifted to have its first peak overlapped with that of m-THF solution), a slight difference in the red side band of the PL spectra was observed. This implies that there is still a little amount of aggregated species in the $\mathrm{C} 0 \mathrm{~m}$-THF solution of high molecular weight. In m-THF solutions, even for the lowest concentration $\mathrm{C} 0$, we cannot rule out the possibility of aggregation. On the other hand, on the basis of concentration studies, it is clear that, in the chloroform solution, there is only single-chain emission. Thus, it is more reliable to use a shift-and-subtract procedure to obtain aggregate emission. In addition, to verify that selfabsorption does not a play significant role in this study, Rhodamine 610 (which shows a large spectral overlap in absorbance and PL spectra) was diluted in ethanol to three concentrations (COR, $\mathrm{C} 1 \mathrm{R}$, and $\mathrm{C} 2 \mathrm{R})$ that optically matched with $\mathrm{C} 0, \mathrm{C} 1$, and $\mathrm{C} 2 \mathrm{DO}-\mathrm{PPV} / \mathrm{m}-\mathrm{THF}$ solutions, respectively. Although corresponding spectra are not shown here for the sake of brevity, it suffices to state that the normalized absorbance spectra of all three Rhodamine solutions were similar. Furthermore, the normalized PL spectra (excited at $500 \mathrm{~nm}$ ) for the two further diluted solutions are the same. Even in the case of $\mathrm{C} 2 \mathrm{R}$ (with a 10-fold increase in concentration as compared to that of $\mathrm{C} 1 \mathrm{R}$ ), the normalized PL spectrum red-shifted only slightly (by ca. $3 \mathrm{~nm}$ ). This indicates that self-absorption and inner filter-induced spectral distortion were basically negligible, with only minor changes at concentrations greater than C1R.

Aggregation of DO-PPV chains in $\mathrm{m}$-THF is further supported by values of the hydrodynamic radius obtained by laser light scattering for 16 and $9 \mathrm{kDa} \mathrm{DO}-\mathrm{PPV}$ in various solvents with a concentration of $10^{-4} \mathrm{M}$. Results show that the $\mathrm{z}$-average hydrodynamic radius for $16 \mathrm{kDa} \mathrm{DO}-\mathrm{PPV}$ in $\mathrm{m}-\mathrm{THF}$ (a poor solvent) is $1100 \pm 600 \mathrm{~nm}$, much greater than that in chloroform (a good solvent) of $18 \pm 20 \mathrm{~nm}$. For $9 \mathrm{kDa}$ DO$\mathrm{PPV}$, the hydrodynamic radius was $14 \pm 30 \mathrm{~nm}$ both in $\mathrm{m}-\mathrm{THF}$ and in chloroform. The dramatic increase in hydrodynamic radius points to aggregation of $16 \mathrm{kDa} \mathrm{DO}-\mathrm{PPV}$ chains in the poor solvent.

Optical Properties at $77 \mathrm{~K}$. At room temperature, the aggregate emission is often significantly lower in efficiency as compared to the single-chromophore path. The presence of competing emitting paths of different quantum yields complicates the analysis of time-resolved fluorescence decay. The fluorescence quantum yield of DO-PPV at low temperature is much higher than that at room temperature, ${ }^{47}$ indicating suppression of nonradiative decay. The dominance of radiative 


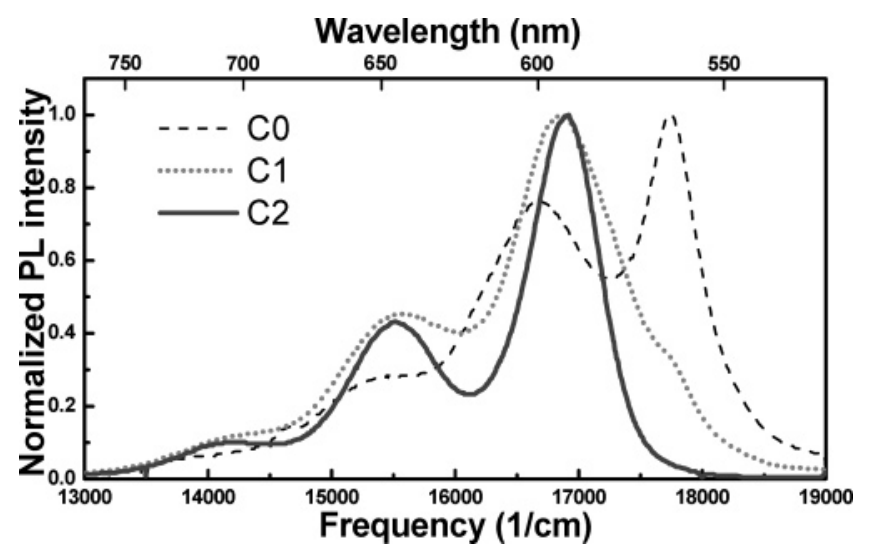

Figure 4. Photoluminescence spectra of DO-PPV in m-THF at $77 \mathrm{~K}$ with concentrations of $\mathrm{C} 0=10^{-6}, \mathrm{C} 1=10^{-5}$, and $\mathrm{C} 2=10^{-4} \mathrm{M}$. The excitation wavelength was $420 \mathrm{~nm}$.

processes in low-temperature time-resolved fluorescence decay provides a better opportunity to resolve single-chain and aggregate emissions. At liquid nitrogen temperature, m-THF solution becomes glassy, allowing for low-temperature spectroscopic analysis.

Figure 4 displays the PL spectra excited at $420 \mathrm{~nm}$ for 16 $\mathrm{kDa}$ DO-PPV/m-THF solutions at $77 \mathrm{~K}$. The red shift with increasing concentration resembles that observed at room temperature. From these low-temperature PL data, one may further deduce that there exist two predominant species with spectral peaks separated by approximately $900 \mathrm{~cm}^{-1}$. The dilute sample C0 shows a narrow emission peak near $17750 \mathrm{~cm}^{-1}$, representing the low-temperature single-chain spectrum. The emission is dominated by the aggregated emission at the highest concentration $\mathrm{C} 2$. For the intermediate case of $\mathrm{C} 1$, the spectrum is a linear combination of the two extremes. Although the excitation wavelength $(420 \mathrm{~nm})$ is distant from the absorbance range of aggregates, energy is transferred from single-chain excitons to the lower-energy aggregated state. It is noted that the same tendency toward formation of interchain species with increasing concentration was observed for solutions at room temperature and at $77 \mathrm{~K}$. We note that the ratio of the aggregates produced by adjusting the poor solution will be effected by the preparation method, conditions, and temperature. In particular, at the lower temperature of $77 \mathrm{~K}, \mathrm{~m}$-THF becomes an even poorer solvent than the room-temperature counterpart; hence, the proportion of aggregated/single-chain increases. That is why the spectra in Figure 3a (at room temperature) do not change as dramatically as those in Figure 4 (at $77 \mathrm{~K}$ ).

Given in Figure 5a are the low-temperature fluorescence decays monitored at $17700 \mathrm{~cm}^{-1}(565 \mathrm{~nm})$ for 9 and $16 \mathrm{kDa}$ DO-PPV in $\mathrm{m}$-THF at concentration C0. The fluorescence decay curves of 9 (open triangles) and $16 \mathrm{kDa}$ DO-PPV (open circles) in $\mathrm{m}$-THF at concentration $\mathrm{C} 0$ were fitted using a singleexponential decay with decay times of $0.979 \pm 0.03\left(\chi^{2}=\right.$ $0.99527)$ and $0.966 \pm 0.03 \mathrm{~ns}\left(\chi^{2}=0.99552\right)$, respectively. Both curves exhibit the same lifetime of $\sim 1.0 \mathrm{~ns}$ under a laser excitation of $500 \mathrm{~nm}$. This is consistent with the dominance of single-chromophore emission deduced from the steady-state PL results, which, in turn, means that the lifetime of singlechromophore emission is $1.0 \mathrm{~ns}$. Shown in Figure $5 \mathrm{~b}$ are fluorescence decays ( $\lambda_{\mathrm{ex}}=500$ or $560 \mathrm{~nm}$ ) monitored at 16800 $\mathrm{cm}^{-1}(595 \mathrm{~nm})$ for different concentrations of $16 \mathrm{kDa} \mathrm{DO}-$ PPV in m-THF. The fluorescence decay curves of $16 \mathrm{kDa}$ DOPPV in m-THF at concentration $\mathrm{C} 2$, which were excited at 500 (dotted line) and 560 (dashed line) nm (monitored at 16800 $\mathrm{cm}^{-1}$ ), were fitted using single-exponential decay with decay
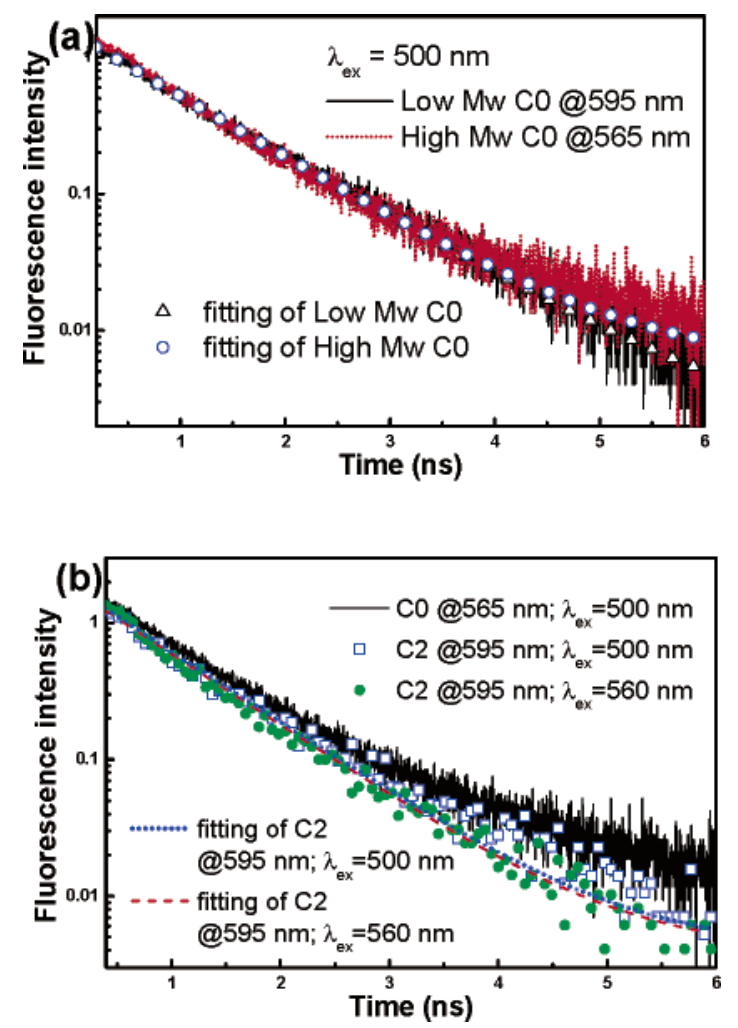

Figure 5. Low-temperature ( $77 \mathrm{~K})$ time-resolved fluorescence decay curves excited at $\lambda_{\mathrm{ex}}=500 \mathrm{~nm}$ (unless specified otherwise) and monitored at (@) the first emission peak (a) for 16 (@565 nm) and 9 kDa (@595 nm) DO-PPV in m-THF at a concentration of C0. The open triangles and the open circles are the fitting results of $9 \mathrm{kDa}$ (with decay time of $0.979 \pm 0.03 \mathrm{~ns}, \chi^{2}=0.99527$ ) and $16 \mathrm{kDa}$ (with decay time of $0.966 \pm 0.03 \mathrm{~ns}, \chi^{2}=0.99552$ ), respectively. (b) The $16 \mathrm{kDa}$ DO-PPV in m-THF at concentrations of C0 (solid line, @565 nm) and C2 (open squares, @595 nm). Filled circles correspond to the case of $\mathrm{C} 2$ excited at $\lambda_{\mathrm{ex}}=560 \mathrm{~nm}$ (@595 nm). The dotted line and the dashed line are the fitting results of the $\mathrm{C} 2$ concentration excited at 500 (with decay time of $0.835 \pm 0.03 \mathrm{~ns}, \chi^{2}=0.99220$ ) and $560 \mathrm{~nm}$ (with decay time of $0.826 \pm 0.03 \mathrm{~ns}, \chi^{2}=0.97854$ ), respectively.

times of $0.835 \pm 0.03\left(\chi^{2}=0.99220\right)$ and $0.826 \pm 0.03 \mathrm{~ns}\left(\chi^{2}\right.$ $=0.97854)$, respectively. A lifetime of $\sim 0.8 \mathrm{~ns}$ describes the two $\mathrm{C} 2$ curves, irrespective of the difference in excitation energy. We emphasize that this difference between the two lifetime values is significant in the corresponding semilogarithmic plots, as all measurements were made in a consistent manner. Since excitation at $560 \mathrm{~nm}$ is expected to excite only the aggregates, Figure 5b signifies the dominance of aggregate emission at concentration $\mathrm{C} 2$; this is consistent with steadystate PL results in Figure 4.

Extended Conjugation. Up to the present point, we have used the term aggregate emission in the very broad sense that it corresponds to the presence of a stable ground state that is directly excitable. One simple interpretation of the red-shifted aggregate emission is that it is a result of extended conjugation length for tighter molecular packing upon aggregation; yet, it involves no interchain delocalization. For a dissolved chain, backbone conjugation is subject to disruption due to torsional motion; hence, an oligomer with finite chain length serves as an appropriate model. ${ }^{48}$ The tighter packing in aggregates (formed due to either decreased solvency power or increased concentration) tends to limit torsional motion, thereby increasing the conjugation length.

On the basis of the exciton model and semiempirical calculation, ${ }^{48,49}$ the energy of the excited state of oligomer depends on the conjugation length $N$ through $E(N)=E_{0}-$ 
$2 \beta \cos [\pi /(N+1)]$ with $E_{0} \sim 4.3 \mathrm{eV}$ and $\beta \sim 1.1 \mathrm{eV}$. According to this formula, the PL spectrum of the dilute solution is mainly attributed to the conjugation length $N=6$ at room temperature. The energy of the new emission band is lower than that of the intrachain state by $1500 \mathrm{~cm}^{-1}$ (from 18600 to $17100 \mathrm{~cm}^{-1}$ ) at room temperature and $1800 \mathrm{~cm}^{-1}$ at low temperature. The shift of the aggregated band will correspond to the shift from $N=6$ to infinite conjugated length, according to the exciton model. Thus, we would expect that the radiative lifetime of the aggregate state will be extremely short due to the large dipole moment. However, the low-temperature time-resolved PL data show that the excited-state lifetime of the aggregates is indeed shorter than that of single-chain excitons (1.0 vs $0.8 \mathrm{~ns})$, but the difference is, by no means, dramatic. Therefore, we believe that the conjugation length increase is basically not the reason leading to the formation of red-band PL in Figure 3b. Nevertheless, the low-temperature PL spectrum for the extremely diluted solution $\mathrm{C} 0$ in Figure 4 might possibly manifest some chain length effect; the red shift in the peak positions from room temperature $\left(18700 \mathrm{~cm}^{-1}\right.$ in Figure 3a) to low temperature (17750 $\mathrm{cm}^{-1}$ in Figure 4) is about $950 \mathrm{~cm}^{-1}$, which corresponds to an increase in conjugation length from $N=6$ at room temperature to $N=10$ at $77 \mathrm{~K}$. It should be noted that the present argument ignores the possibility that coupling between monomers can change as the surrounding environment changes.

Interchain Delocalization. An alternative model is the dimer formation. For a ground-state bound dimer, the two extreme cases are $\mathrm{H}$ and $\mathrm{J}$ aggregates. For $\mathrm{H}$ aggregates, the lowest excited state is dipole forbidden because it corresponds to two dipoles which are antiparallel to each other; thus, the quantum yields for light emission from the lowest excited state should be extremely low. In the case of the J aggregates, the lowest excite state corresponds to two parallel dipoles falling in line; the lowest excited-state is thus dipole allowed and has high quantum efficiency for light emission. As the single-exciton quantum yield of the aggregate in DO-PPV is only moderately lower than that of the single-chain exciton ${ }^{47}$ the possibility of strict $\mathrm{H}$-type aggregates is ruled out. On the other hand, the length of the intrachain exciton suggests that strict J-type aggregation is also quite unlikely. Thus, if morphological aggregation results in the formation of delocalized dimeric species, these should be intermediate to idealized $\mathrm{H}$ and $\mathrm{J}$ aggregates. This picture is supported by the ab inito calculation of $\mathrm{Yu}$ et al., ${ }^{49}$ who analyzed the excited-state properties of two molecules of PPV hexamers separated by $0.3 \mathrm{~nm}$ and longitudinally shifted by $0.35 \mathrm{~nm}$. They found that the lowest excited state in this dimer is red-shifted with respect to the single-chain hexamer and, more importantly, has an oscillator strength similar to that of the single-chain hexamer.

Consistent with the discussion above, our low-temperature results in Figure 5b show that single-chain and aggregate excitons have similar decay lifetimes. Since the decay lifetime is dominated by the radiative process at low temperatures, it is directly related to the oscillator strength and radiative decay time; we may, hence, assume that there are mainly two emissive species. According to the previous discussion, these correspond to intrachain and aggregate states. In a previous publication, we have demonstrated that a two-state model can describe roomtemperature absorbance, PL, and decay dynamics. ${ }^{46}$ In the present low-temperature case, PL spectra in the three concentrations were simulated by combining the two emissive spectral profiles. Calculated spectral profiles $I_{\mathrm{m}}$ and $I_{\mathrm{a}}$ of intrachain and aggregate states, respectively, at $77 \mathrm{~K}$ are shown in Figure 6. The vibronic structure in the profiles of $I_{\mathrm{m}}$ and $I_{\mathrm{a}}$ are more
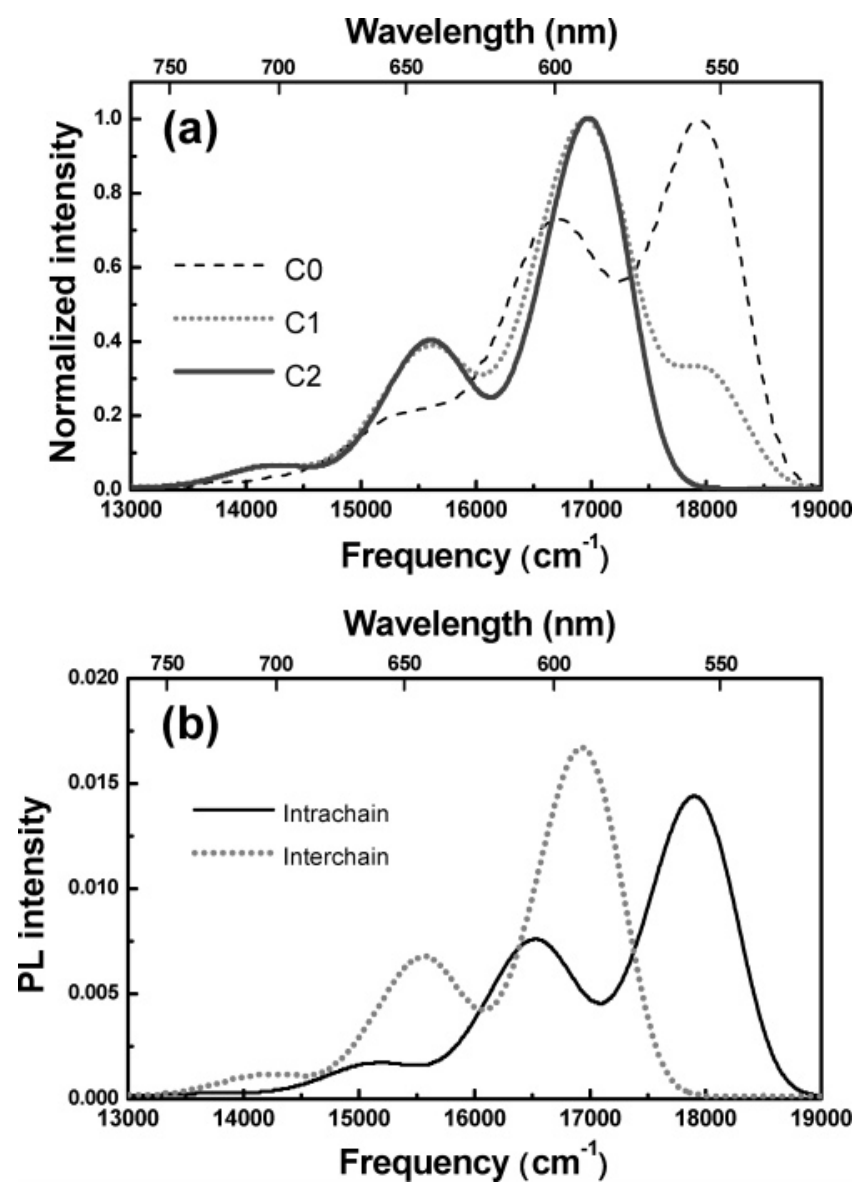

Figure 6. (a) Calculated photoluminescence spectral profiles for intrachain and interchain states at low temperature. (b) Calculated photoluminescence spectra for concentrations $\mathrm{C} 0, \mathrm{C} 1$, and $\mathrm{C} 2$ at low temperature.

evident because the thermal energy of low-frequency modes is effectively suppressed at low temperature. The peak position of $I_{\mathrm{m}}(\omega)$ is shifted from the room-temperature case by $950 \mathrm{~cm}^{-1}$ due to elongation of the conjugation length. As discussed earlier, this corresponds to a conjugation length of ca. 10 repeating units; a further increase in chain length will not result in significant changes in energy. Thus, the appearance of a second peak is assigned to the aggregate state. The ratio of intensities of singlechain versus aggregate emissions is 1:1 in Figure 6a. The C0 profile is dominated by single-chromophore emission with $I_{\mathrm{a}} /$ $I_{\mathrm{m}}=1: 5.36$; the contribution of $I_{\mathrm{a}}$ and $I_{\mathrm{m}}$ in the C1 case is $I_{\mathrm{a}} / I_{\mathrm{m}}$ $=1: 0.42$; in the case of $\mathrm{C} 2$, the emission is all dominated by dimeric aggregates, where $I_{\mathrm{a}} / I_{\mathrm{m}}=1: 0$.

Results of single-molecule spectroscopy of MEH-PPV have demonstrated that, within the inert poly(methyl methacrylate) host matrix, isolated MEH-PPV chains exhibit two distinctive fluorescence spectra of clear vibronic structure; the leading emission maxima are located at wavelengths of 553 and 583 $\mathrm{nm} .{ }^{50}$ This corresponds to a difference of ca. $900 \mathrm{~cm}^{-1}$, whereas the low-energy emission band at $583 \mathrm{~nm}$ was assigned to aggregates originating from the chain-chain contacts within a single polymer. ${ }^{51}$ All of these are consistent with the present results.

Theoretical Calculation of Aggregate Spectra. To analyze the observed absorbance and fluorescence spectra from dimeric aggregates, we use the displaced-distorted harmonic potential energy surface model within the Frank-Condon and the BornOppenheimer approximations. ${ }^{52}$ In this case, the absorption coefficient, a function of absorption frequency $\omega$, is given by 
the generating function method as

$$
\begin{aligned}
&\langle\alpha(\mathrm{i} \rightarrow \mathrm{f})\rangle_{\mathrm{av}}=k \omega\left|\mu_{\mathrm{fi}}(0)\right|^{2} \int_{-\infty}^{\infty} \mathrm{d} t \exp \left[i t\left(\bar{\omega}_{\mathrm{fi}}-\omega\right)-\right. \\
&\left.\Delta^{2} t^{2} / 4-|t| \gamma_{\mathrm{fi}}\right] \prod_{j=1}^{N} G_{j}(t)
\end{aligned}
$$

where $k=2 \pi / 3 \hbar n_{\mathrm{e}}(\omega) c$ and $n_{\mathrm{e}}(\omega)$ is the refractive index, $\mu_{\mathrm{fi}}(0)$ is the transition dipole moment from the ground (i) to the excited (f) states, $\bar{\omega}_{\mathrm{fi}}$ is the averaged $0-0$ transition energy between states $\mathrm{i}$ and $\mathrm{f}$ in the solvent environments, $\Delta$ is the inhomogeneity of the electronic transition energy by using the Gaussian distribution function, $\gamma_{\mathrm{fi}}$ the electronic dephasing constant, and $N$ the number of vibrational modes, whereas $G_{j}(t)$, the nuclear correlation function of the $j$ th vibrational normal mode, is given by

$$
\begin{aligned}
& G_{j}(t)=\frac{2 n_{j}^{\prime} \mathrm{e}^{i t\left(\omega_{j}-\omega_{j}^{\prime \prime}\right) / 2}\left(\mathrm{e}^{\hbar \omega_{j} / k T}-1\right)}{\omega_{j}+\omega_{j}^{\prime \prime}} \sqrt{\frac{\omega_{j} \omega_{j}^{\prime \prime}}{f_{j}^{+} f_{j}^{-}}} \times \exp \\
& \left\{\frac{-\omega_{j} \omega_{j}^{\prime \prime} d_{j}^{2}}{\hbar\left(\omega_{j}+\omega_{j}^{\prime \prime}\right)} \times \frac{\left(1+2 n_{j}^{\prime}\right)-\left(n_{j}^{\prime}+1\right) \mathrm{e}^{i t \omega_{j}^{\prime \prime}}-n_{j}^{\prime} \mathrm{e}^{-i t \omega_{j}^{\prime \prime}}}{f_{j}^{-}}\right\}
\end{aligned}
$$

in which $f_{j}^{ \pm}=1 \pm\left[\left(\omega_{j}^{\prime \prime}-\omega_{j}\right) /\left(\omega_{j}^{\prime \prime}+\omega_{j}\right)\right]\left\{\left(n_{j}^{\prime}+1\right) \mathrm{e}^{i t \omega_{j}^{\prime \prime}}-\right.$ $\left.n_{j}^{\prime} \mathrm{e}^{-i t \omega_{j}^{\prime \prime}}\right\}, n_{j}^{\prime}=\left[\mathrm{e}^{i t\left(\omega_{j}-\omega_{j}^{\prime \prime}\right)+\hbar \omega_{j} / k T}-1\right]^{-1}, \omega_{j}$ and $\omega_{j}^{\prime \prime}$ are vibrational frequencies of the $j$ th vibrational mode at states $i$ and $\mathrm{f}$, respectively, and $d_{j}$ is the displacement between states $\mathrm{i}$ and $\mathrm{f}$ in the normal coordinates of the $j$ th mode. The fluorescence coefficient $\left\langle\epsilon(\mathrm{f} \rightarrow \mathrm{i}\rangle_{\mathrm{av}}\right.$, a function of the emission frequency $\omega$, is then given by

$$
\begin{aligned}
\langle\epsilon(\mathrm{f} \rightarrow \mathrm{i})\rangle_{\mathrm{av}}=k^{\prime} \omega^{3}\left|\bar{\mu}_{\mathrm{if}}(0)\right|^{2} \int_{-\infty}^{\infty} \mathrm{d} t \exp \left[i t\left(\omega-\bar{\omega}_{\mathrm{fi}}\right)-\right. \\
\left.\Delta^{2} t^{2} / 4-|t| \gamma_{\mathrm{fi}}\right] \prod_{j=1}^{N} G_{j}(t)
\end{aligned}
$$

where $k^{\prime}=2 n_{\mathrm{e}}(\omega) / 3 c^{2} A_{\mathrm{fi}}$ and $A_{\mathrm{fi}}$ is the radiative constant from the states $\mathrm{f}$ to $\mathrm{i}$ and the transition dipole moment $\mu_{\mathrm{if}}(0)=\mu_{\mathrm{fi}}(0)$. Note that $G_{j}(t)$ in $\left\langle\epsilon(\mathrm{f} \rightarrow \mathrm{i}\rangle_{\text {av }}\right.$ can be obtained by exchanging $\omega_{j}$ and $\omega_{j}^{\prime \prime}$ in eq 2 . In this case, the absorbance and emission spectra can be characterized and are proportional to $\langle\alpha(\mathrm{i} \rightarrow \mathrm{f})\rangle_{\mathrm{av}} / k \omega\left|\mu_{\mathrm{fi}}(0)\right|^{2}$ and $\langle\epsilon(\mathrm{f} \rightarrow \mathrm{i})\rangle_{\mathrm{av}} / k^{\prime} \omega^{3}\left|\mu_{\mathrm{if}}(0)\right|^{2}$, respectively.

In the analysis, we first focus on the absorbance and fluorescence spectra of DO-PPV single chains. In this case, because of the similar chemical structure of the backbone and vibrational spectra between DO-PPV and MEH-PPV, we adopt the vibrational frequencies of the ground state according to the previous work on MEH-PPV. ${ }^{48}$ Results of our calculations indicate that vibrational frequencies at 1400, 700, and 200 $\mathrm{cm}^{-1}$ correspond to the exciton ground state, while those at 1250,600 , and $200 \mathrm{~cm}^{-1}$ are for the exciton excited state. In the potential energy surface, maximum displacements of the corresponding vibrational modes were $0.27,0.43$, and 1.5 $(\mathrm{amu})^{1 / 2} \AA$, whereas the inhomogeneous broadening and averaged $0-0$ transition energies were 450 and $17670 \mathrm{~cm}^{-1}$, respectively. The spectrum of aggregated DO-PPV could be obtained from the discrepancy between the absorbance spectra of DO-PPV dissolved in m-THF (contains single-chain and aggregated components) and chloroform (single-chain only), as shown in Figure 3b. The aggregate absorbance spectrum is extracted by spectral deconvolution shown in Figure 7. The basic idea of the deconvolution is the following. At $\mathrm{C} 2$ concentration, the absorbance spectrum includes both single-chain as well as aggregated absorption. Therefore, first, three Gaussian functions are used to simulate the absorbance spectrum of high molecular

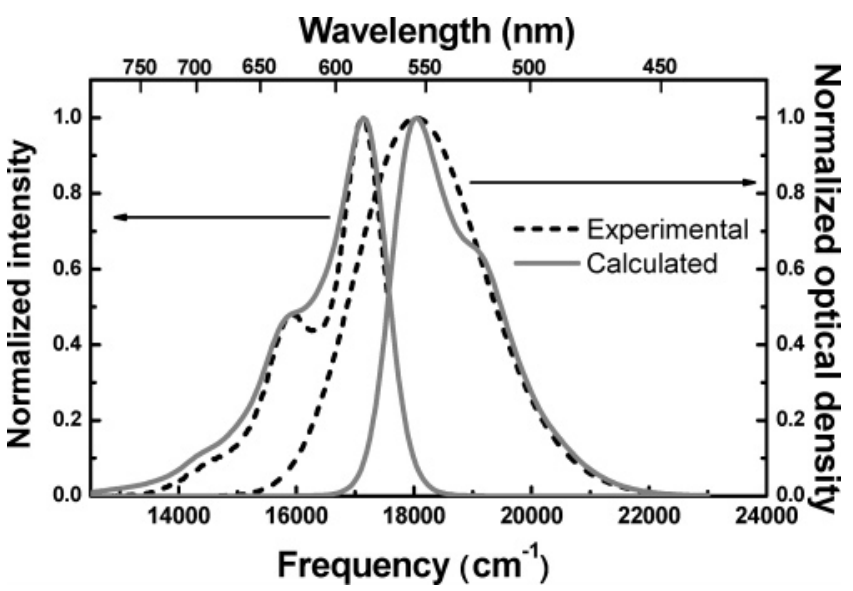

Figure 7. Experimental (dashed line) and calculated (solid line) absorbance and photoluminescence spectra of DO-PPV aggregates.

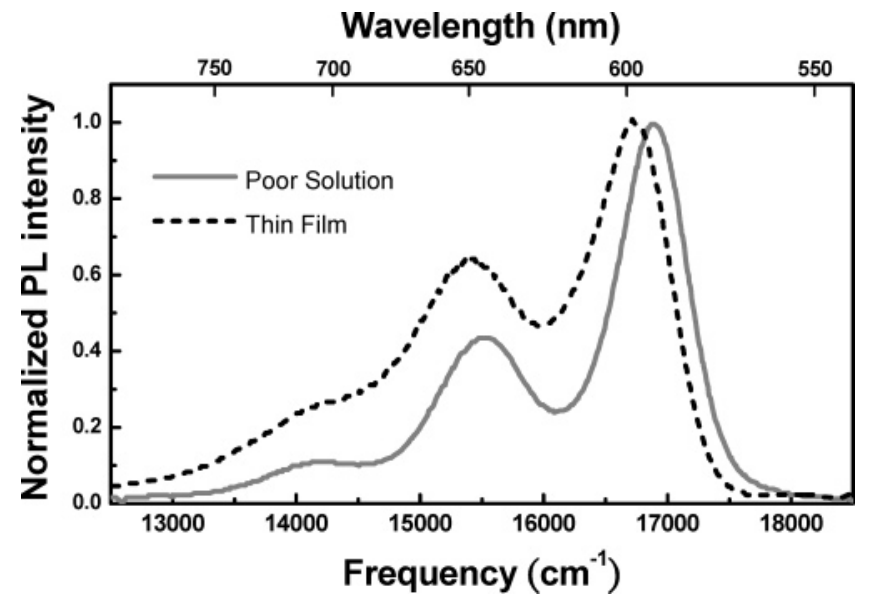

Figure 8. Photoluminescence spectra for the thin film and solution $\mathrm{C} 2$ at $77 \mathrm{~K}$.

weight DO-PPV in good solvent, in which single-chain absorption only is expected. Next, the relative amplitudes and widths of these three Gaussian functions are used as the basis to fit the absorbance in C2 (high concentration in poor solvent) with one additional Gaussian function representing the aggregated absorbance. Considering effects from difference dielectric constants, we have allowed the central positions of the three Gaussian functions to move slightly from good to poor solvent. Commercial software (PeakFit, version 4, SPSS Inc.) was used to search the best aggregated Gaussian function. Figure 7 shows the calculated spectrum and the measured aggregated absorbance spectrum. Note that the PL spectrum excited at 560 $\mathrm{nm}$ in Figure $3 \mathrm{~b}$ is similar to the aggregate PL spectrum from spectral subtraction here. This validates the calculated aggregate spectrum that gives the averaged $0-0$ transition energy as 17670 $\mathrm{cm}^{-1}(566 \mathrm{~nm})$.

Luminescence from Thin Films. Films prepared by either spin coating or drop casting may be considered as the limit of increasingly concentrated solutions. In view of the high density of interchain contacts in films, one expects weaker chain length effects in aggregate formation and more efficient energy transfer from single-chromophore to aggregate excitons. Thin films were prepared via spin casting using a DO-PPV sample with $M_{\mathrm{w}}=$ ca. $10 \mathrm{kDa}$. The low-temperature emission spectra, shown in Figure 8, resemble that of the low-temperature emission spectrum of $\mathrm{C} 2$ in Figure 4, qualitatively confirming that the emission from films is dominantly from aggregates. Quantitatively, there exist minor differences. The slight shift of the peak position between the film and $\mathrm{C} 2$ can be attributed to the 

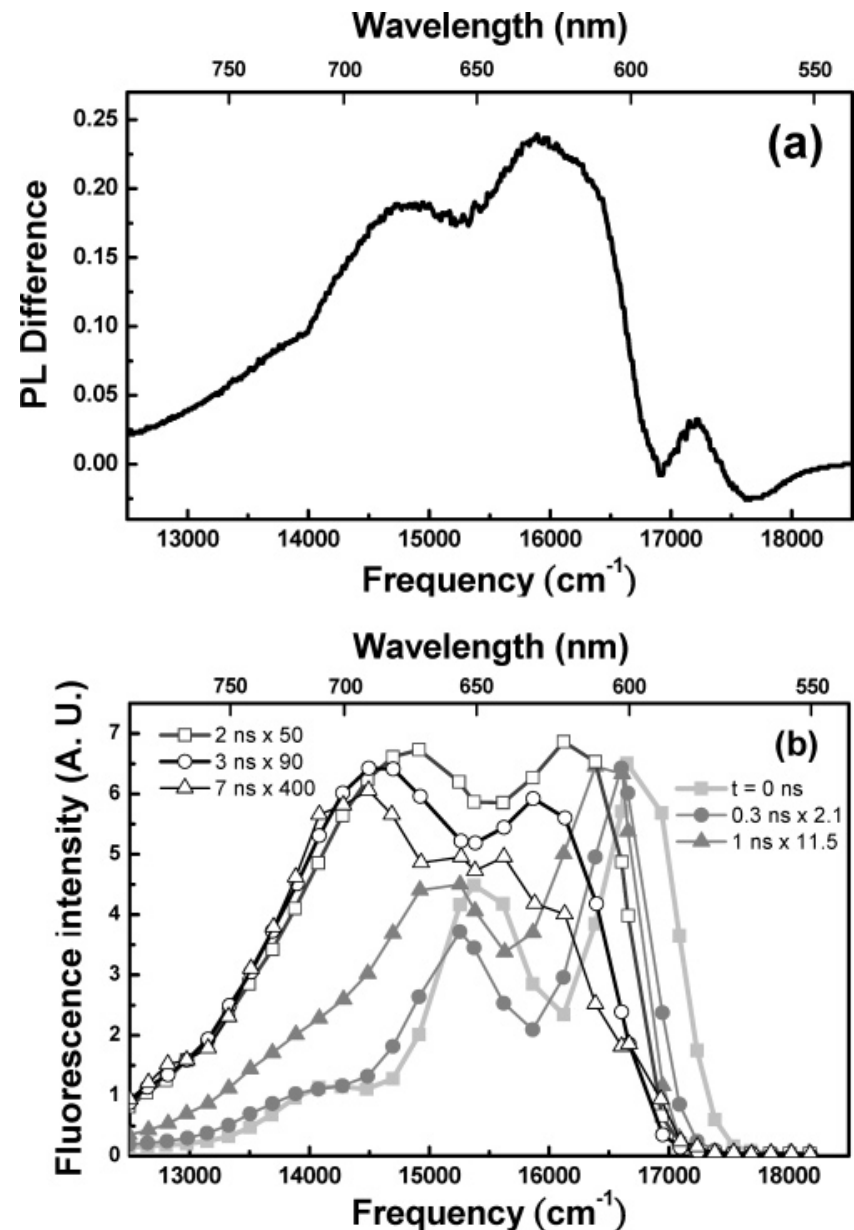

Figure 9. (a) Difference spectrum between the thin film and solution C2 obtained from the two spectra in Figure 8. (b) Time-resolved photoluminescence spectra of the thin film at different times. Note the similarity between the spectra at longer delay times ( 3 and $7 \mathrm{~ns}$ ) with that in (a).

difference in dielectric constants of the film and m-THF. More importantly, as shown in Figure 9a, the difference spectrum between the film and $\mathrm{C} 2$ (obtained by a shift-and-subtract procedure, where the aggregate spectrum was shifted to have its leading peak overlapping with that of the film spectrum) indicates the presence of a low-energy featureless emission in the film state. This type of minor emission at the red end is often attributed to long lifetime entities such as interchain-bound polaron pairs or excimers, which have been shown to exist in thin films of conjugated polymers. Specifically, excimer emission at $16000 \mathrm{~cm}^{-1}$ has been proposed for aggregated DOPPV in a xylene solution. ${ }^{53}$

As shown in Figure 9b, the excimer assignment is supported by low-temperature time-resolved fluorescence spectra of thin films. Within 0 to $1 \mathrm{~ns}$, a spectral shape similar to that in the emission spectrum of solution $\mathrm{C} 2$ in Figure 8 can be identified. The lifetime is about $0.3 \mathrm{~ns}$, shorter than that in the poor solution C2 case, $0.8 \mathrm{~ns}$. A new fluorescence band starts to appear at ca. 3 ns beyond pulsed excitation. This new emission band that appeared at a long delay time has a similar shape and peak position as those of the difference spectrum in Figure 9a, implying a lifetime longer than $3.0 \mathrm{~ns}$ for the low-energy state, which is consistent with the assignment of excimeric emission. In view of the clear decrease (from 0.8 to $0.3 \mathrm{~ns}$ ) in the apparent lifetime of aggregate emission, there must be efficient energy transfer between the aggregate and excimer states.
We may, therefore, conclude that there exist two emission species, the aggregates and the excimers, in the thin film at 77 $\mathrm{K}$. The emission from the aggregated state exhibits clear vibronic structure, while the emission from the excimer is broad and featureless. Previously, Samuel et al. ${ }^{32-34}$ identified an excimeric state with a lifetime of $\sim 5.0 \mathrm{~ns}$ (corresponding to a radiative decay time of $\sim 16 \mathrm{~ns}$ ) in CN-PPV. Jakubiak et al. ${ }^{39}$ observed an excimeric state with a lifetime of $\sim 425 \mathrm{~ns}$ in MEH-PPV at a cryogenic temperature. Although decay rates are different, the states observed by both groups have similar spectral profiles centered at $\sim 14000 \mathrm{~cm}^{-1}$, which are also very close to the $7 \mathrm{~ns}$ profile in Figure 9b.

\section{Conclusion}

In summary, we have examined spectral properties of the aggregate state by varying solution concentration and solvent quality for two fractions of DO-PPV of different chain lengths both at ambient and liquid nitrogen temperatures. The same aggregate state is the main emission species in thin films. An additional low-energy excitonic state was identified for films at low temperature, which bears similar spectral characteristics previously ascribed to excimers in the case of $\mathrm{CN}-\mathrm{PPV}$ and $\mathrm{MEH}-\mathrm{PPV}$, two close homologues of DO-PPV.

Acknowledgment. Financial support from the Ministry of Education (Grant Number 91-E-FA04-2-4A) is gratefully acknowledged.

\section{References and Notes}

(1) Rauscher, U.; Bassler, H.; Bradley, D. D. C.; Hennecke, M. Phys. Rev. B 1990, 42, 9830 .

(2) Kiess, H. G.; Harbeke, G. Conjugated Conducting Polymers; Springer-Verlag: Berlin, Germany, 1992; Vol. 102

(3) Heun, S.; Mahrt, R. F.; Greiner, A.; Lemmer, U.; Bassler, H.; Halliday, D. A.; Bradley, D. D. C.; Burn, P. L.; Holmes, A. B. J. Phys. Condens. Matter 1993, 5, 247

(4) Lee, C. H.; Yu, G.; Heeger, A. J. Phys. Rev. B 1993, 47, 15543.

(5) Yan, M.; Rothberg, L.; Hsieh, B. R.; Alfano, R. R. Phys. Rev. B 1994, 49, 9419.

(6) Yan, M.; Rothberg, L. J.; Papadimitrakopoulos, F.; Galvin, M. E.; Miller, T. M. Phys. Rev. Lett. 1994, 72, 1104

(7) Greenham, N. C.; Samuel, I. D. W.; Hayes, G. R.; Phillips, R. T.; Kessener, Y. A. R. R.; Moratti, S. C.; Holmes, A. B.; Friend, R. H. Chem Phys. Lett. 1995, 241, 89.

(8) Yan, M.; Rothberg, L. J.; Kwock, E. W.; Miller, T. M. Phys. Rev Lett. 1995, 75, 1992. R2308.

(9) Conwell, E. M.; Perlstein, J.; Shaik, S. Phys. Rev. B 1996, 54,

(10) Harrison, N. T.; Hayes, G. R.; Phillips, R. T.; Friend, R. H. Phys. Rev. Lett. 1996, 77, 1881.

(11) Rothberg, L. J.; Yan, M.; Papadimitrakopoulos, F.; Galvin, M. E.; Kwock, E. W.; Miller, T. M. Synth. Met. 1996, 80, 41.

(12) Klimov, V. I.; McBranch, D. W.; Barashkov, N. N.; Ferraris, J. P. Chem. Phys. Lett. 1997, 277, 109.

(13) Zeng, Q. G.; Ding, Z. J.; Ju, X.; Zhang, Z. M. Eur. Polym. J. 2005, 41,743

(14) Cossiello, R. F.; Akcelrud, L.; Atvars, D. Z. J. Braz. Chem. Soc $2005,16,74$.

(15) Cossiello, R. F.; Kowalski, E.; Rodrigues, P. C.; Akcelrud, L.; Bloise, A. C.; deAzevedo, E. R.; Bonagamba, T. J.; Atvars, T. D. Z Macromolecules 2005, 38, 925 .

(16) Barbara, P. F.; Gesquiere, A. J.; Park, S. J.; Lee, Y. J. Acc. Chem Res. 2005, 38, 602

(17) Scholes, G. D.; Rumbles, G. Nat. Mater. 2006, 5, 683.

(18) Nguyen, T. Q.; Doan, V.; Schwartz, B. J. J. Chem. Phys. 1999, 110,4068

(19) Harre, K.; Wegner, G. Polymer 2006, 47, 7312

(20) Zaroslov, Y. D.; Gordeliy, V. I.; Kuklin, A. I.; Islamov, A. H.; Philippova, O. E.; Khokhlov, A. R.; Wegner, G. Macromolecules 2002 $35,4466$.

(21) Kulkarni, A. P.; Jenekhe, S. A. Macromolecules 2003, 36, 5285

(22) Kulkarni, A. P.; Kong, X. X.; Jenekhe, S. A. J. Phys. Chem. B 2004, 108, 8689 . 
(23) Nguyen, T. Q.; Schwartz, B. J.; Schaller, R. D.; Johnson, J. C.; Lee, L. F.; Haber, L. H.; Saykally, R. J. J. Phys. Chem. B 2001, 105, 5153 (24) Schaller, R. D.; Snee, P. T.; Johnson, J. C.; Lee, L. F.; Wilson, K. R.; Haber, L. H.; Saykally, R. J.; Nguyen, T. Q.; Schwartz, B. J. J. Chem. Phys. 2002, 117, 6688.

(25) Schaller, R. D.; Lee, L. F.; Johnson, J. C.; Haber, L. H.; Saykally, R. J.; Vieceli, J.; Benjamin, I.; Nguyen, T. Q.; Schwartz, B. J. J. Phys. Chem. B 2002, 106, 9496.

(26) Jenekhe, S. A.; Chen, X. L. J. Phys. Chem. B 2000, 104, 6332.

(27) Gettinger, C. L.; Heeger, A. J.; Drake, J. M.; Pine, D. J. J. Chem. Phys. 1994, 101, 1673.

(28) Guha, S.; Graupner, W.; Resel, R.; Chandrasekhar, M.; Chandrasekhar, H. R.; Glaser, R.; Leising, G. Phys. Rev. Lett. 1999, 82, 3625

(29) Gruner, J.; Hamer, P. J.; Friend, R. H.; Huber, H. J.; Scherf, U.; Holmes, A. B. Adv. Mater. 1994, 6, 748.

(30) Jenekhe, S. A.; Osaheni, J. A. Science 1994, 265, 765.

(31) Lemmer, U.; Heun, S.; Mahrt, R. F.; Scherf, U.; Hopmeier, M.; Siegner, U.; Gobel, E. O.; Mullen, K.; Bassler, H. Chem. Phys. Lett. 1995, $240,373$.

(32) Samuel, I. D. W.; Rumbles, G.; Collison, C. J. Phys. Rev. B 1995 , 52,11573

(33) Hayes, G. R.; Samuel, I. D. W.; Phillips, R. T. Phys. Rev. B 1996, 54, R8301

(34) Samuel, I. D. W.; Rumbles, G.; Collison, C. J.; Moratti, S. C.; Holmes, A. B. Chem. Phys. 1998, 227, 75.

(35) Chen, S. H.; Su, C. H.; Su, A. C.; Chen, S. A. J. Phys. Chem. B 2004, 108, 8855 .

(36) Chen, S. H.; Su, A. C.; Huang, Y. F.; Su, C. H.; Peng, G. Y.; Chen, S. A. Macromolecules 2002, 35, 4229 .

(37) Kong, F.; Wu, X. L.; Yuan, R. K.; Yang, C. Z.; Siu, G. G.; Chu, P. K. J. Vac. Sci. Technol., A 2006, 24, 202.

(38) Kong, F.; Wu, X. L.; Huang, G. S.; Yuan, R. K.; Yang, C. Z.; Chu, P. K.; Siu, G. G. Appl. Phys. A 2006, 84, 203.
(39) Jakubiak, R.; Collison, C. J.; Wan, W. C.; Rothberg, L. J.; Hsieh, B. R. J. Phys. Chem. A 1999, 103, 2394.

(40) Narwark, O.; Meskers, S. C. J.; Peetz, R.; Thorn-Csanyi, E.; Bassler, H. Chem. Phys. 2003, 294, 1.

(41) Narwark, O.; Gerhard, A.; Meskers, S. C. J.; Brocke, S.; ThornCsanyi, E.; Bassler, H. Chem. Phys. 2003, 294, 17.

(42) Sarnecki, G. J.; Burn, P. L.; Kraft, A.; Friend, R. H.; Holmes, A. B. Synth. Met. 1993, 55, 914 .

(43) Askari, S. H.; Rughooputh, S. D.; Wudl, F. Synth. Met. 1989, 29 E129.

(44) Hsu, J. H.; Hayashi, M. T.; Lin, S. H.; Fann, W. S.; Rothberg, L. J.; Perng, G. Y.; Chen, S. A. J. Phys. Chem. B 2002, 106, 8582.

(45) Grell, M.; Bradley, D. D. C.; Long, X.; Chamberlain, T.; Inbasekaran, M.; Woo, E. P.; Soliman, M. Acta Polym. 1998, 49, 439.

(46) Chang, R.; Hsu, J. H.; Fann, W. S.; Yu, J.; Lin, S. H.; Lee, Y. Z; Chen, S. A. Chem. Phys. Lett. 2000, 317, 153.

(47) Hsu, J. H.; Fann, W. S.; Tsao, P. H.; Chuang, K. R.; Chen, S. A. J. Phys. Chem. A 1999, 103, 2375.

(48) Chang, R.; Hsu, J. H.; Fann, W. S.; Liang, K. K.; Chiang, C. H.; Hayashi, M.; Yu, J.; Lin, S. H.; Chang, E. C.; Chuang, K. R.; Chen, S. A. Chem. Phys. Lett. 2000, 317, 142.

(49) Yu, J. W.; Fann, W. S.; Kao, F. J.; Yang, D. Y.; Lin, S. H. Synth. Met. 1994, 66, 143 .

(50) Yu, J.; Hu, D. H.; Barbara, P. F. Science 2000, 289, 1327.

(51) Yu, J.; Hu, D. H.; Barbara, P. F. Nobel Conference Lectures; Springer Series in Chemical Physics Vol. 67; Springer: Berlin, Germany, 2001; p 123.

(52) Shiu, Y. J.; Hayashi, M.; Mebel, A. M.; Chen, Y. T.; Lin, S. H. J. Chem. Phys. 2001, 115, 4080.

(53) Chen, S. H.; Su, A. C.; Han, S. R.; Chen, S. A.; Lee, Y. Z. Macromolecules 2004, 37, 181. 